\title{
CUSTEIO ABC NO AMBIENTE HOSPITALAR: UM ESTUDO NOS HOSPITAIS UNIVERSITÁRIOS E DE ENSINO BRASILEIROS*
}

\author{
ABC COSTING IN HOSPITAL ENVIRONMENT: \\ A STUDY IN BRAZILIAN UNIVERSITY HOSPITALS
}

\author{
GILBERTO JOSÉ MIRANDA \\ Professor Mestre \\ da Faculdade de Ciências Integradas do Pontal \\ da Universidade Federal de Uberlândia - MG \\ E-mail: gilberto@pontal.ufu.br \\ CLEVERTON EUCLEN DE CARVALHO \\ Graduando em Ciências Contábeis \\ pela Faculdade de Ciências Contábeis \\ da Universidade Federal de Uberlândia - MG \\ E-mail: clevertonspfc@yahoo.com.br
}

\author{
VIDIGALL FERNANDES MARTINS \\ Professor Mestre \\ da Faculdade de Ciências Contábeis \\ da Universidade Federal de Uberlândia - MG \\ E-mail: vidigal@ufu.br \\ ADOLAR FERREIRA DE FARIA \\ Professor Graduado \\ da Faculdade de Ciências Contábeis \\ da Universidade Federal de Uberlândia - MG \\ E-mail: adoalr@ufu.br
}

\section{RESUMO}

O Custeio Baseado em Atividades tem se mostrado como uma alternativa promissora para fazer frente à complexidade que caracteriza os custos hospitalares. Nos últimos dez anos, somente na Plataforma Lattes, foram encontrados mais de uma centena de estudos dessa natureza. Este trabalho tem como objetivo, conhecer, empiricamente, a utilização do Custeio $\mathrm{ABC}$ nos hospitais universitários e de ensino brasileiros e comparar os resultados, conforme as possibilidades, com as pesquisas realizadas nas maiores empresas brasileiras pelos autores: Khoury (1999), Beuren e Roedel (2002) e Azevedo, Santos e Pamplona (2004). Dos 115 questionários enviados aos hospitais universitários, 34 foram respondidos. O estudo levou a conclusões importantes, como: Os sistemas de custos atuais dos hospitais têm poucas condições de fornecer informações úteis à gestão; o Custeio $\mathrm{ABC}$ é bastante conhecido no ambiente, mas o número de usuários ainda é relativamente pequeno: apenas 15\% da amostra; mas existe expectativa por parte de $44 \%$ dos hospitais com relação ao uso futuro da abordagem. As principais causas apresentadas para a não-utilização do Sistema $A B C$ foram: (a) o sistema utilizado atende às necessidades da organização e (b) o Custeio Baseado em Atividades é muito complexo.

Palavras-chave: Custos; Hospitais; Brasil.

\section{ABSTRACT}

The Cost Based Activity has been a promising alternative to deal with the complexity that characterizes hospital costs. In the last ten years, only in the Plataforma Lattes, more than a hundred studies of this nature had been found. This work aims to find out, empirically, the use of $A B C$ Costing in Brazilian university hospitals and to compare the results, according to the possibilities, with the researches that have been made in the biggest Brazilian companies by the authors: Khoury (1999), Beuren and Roedel (2002) and Azevedo, Santos and Pamplona (2004): A hundred and fifteen questionnaires were sent to the university hospitals, 34 had been answered. The study relates important conclusions: The current cost systems of the hospitals have few conditions to supply useful information to the management; the $A B C$ Costing is enough known in the hospital area, but the number of users is still relatively small: only $15 \%$ of the sample; but there is a expectation on the part of $44 \%$ of the hospitals regarding the future use of the boarding. The main causes presented for not-using the $A B C$ Costing had been: (a) the system in use serves the necessities of the organization and (b) the Activity Based Costing is very complex.

Keywords: Cost; Hospitals; Brazil. 


\section{INTRODUÇÃO}

O Custeio Baseado em Atividades ( $A B C)$ tem sido uma abordagem bastante discutida, nas duas últimas décadas, no cenário brasileiro. A sua utilidade é verificada no apoio ao processo decisório dos empreendimentos, especialmente, em setores em que os custos fixos são elevados. Nesses ambientes, abordagens como o Custeio Variável perdem sua relevância, pois os custos fixos são "ignorados" na composição do custo total dos produtos. O Custeio $A B C$ considera os gastos fixos, mediante o rastreamento das causas que os provocam. Com base nesse atributo, Martins (2003, p. 87) afirma que o "Custeio Baseado em Atividades $(A B C)$ não se limita ao custeio dos produtos. Ele é, acima de tudo, uma poderosa ferramenta a ser utilizada na gestão de custos".

Khoury (1999) apresenta resultados de pesquisas realizadas em outros países com a finalidade de verificar o uso do Custeio $A B C$ no ambiente empresarial. De acordo com esses dados, em 1991, o Custeio Baseado em Atividades estava presente em $6 \%$ das empresas inglesas e em $11 \%$ das americanas; em 1992, em $14 \%$ das empresas canadenses e, em 1993, 36\% das empresas americanas já estavam utilizando o Custeio $A B C$.

Com o intuito de investigar o uso pragmático do Custeio $A B C$ no Brasil, Khoury (1999) fez um levantamento nas quinhentas maiores empresas brasileiras relacionadas na Revista Exame - Melhores e Maiores, no ano de 1996. Obtendo uma amostra de 283 companhias, foi constatado que apenas $18 \%$ utilizavam o Custeio $A B C$. Posteriormente, Azevedo, Santos e Pamplona (2004) reaplicaram a pesquisa também nas quinhentas maiores empresas relacionadas na Revista Exame. Foi identificada uma queda no uso da abordagem, verificando-se que apenas $14,3 \%$ da amostra utilizava essa forma de custeio. Beuren e Roedel (2002) também reaplicaram a pesquisa de Khoury (1999) nas duzentas maiores empresas catarinenses, mas obtiveram um percentual de uso do Custeio Baseado em Atividades menor ainda, apenas $7 \%$ da amostra.

No ambiente hospitalar, a apuração de custos assume singular complexidade em função da heterogeneidade de "produtos" que são oferecidos aos "consumidores", notadamente nos hospitais universitários e de ensino. Pois, esses, além de gerarem serviços à população, em que os procedimentos realizados variam a cada caso, uma vez que as pessoas reagem de formas diferentes ante os problemas de saúde, também formam os futuros profissionais da saúde. Na linguagem contábil, a formação dos profissionais são ativos constituídos ao longo de todo o período escolar e depois oferecidos à sociedade para o exercício das profissões.

Para fazer face a tal complexidade, muitos estudos se fazem necessários. Nesse sentido, a aplicação do Custeio $A B C$ no ambiente hospitalar tem merecido a atenção de diversos pesquisadores. Na Plataforma Lattes, no mês de Agosto/06, foram encontradas 130 publicações de trabalhos envolvendo o Custeio $A B C$ e o ambiente hospitalar. Todos eles realizados nos últimos dez anos, conforme demonstra a Tabela $1 \mathbf{0}$.

Observa-se que, em termos de produção de conhecimento, o Custeio Baseado em Atividades no contexto hospitalar tem tido uma evolução diferente, ou pelo menos retardada se comparada aos estudos anteriormente mencionados. Em 1996, quando Khoury realizou sua pesquisa, foi encontrada apenas uma publicação do Custeio $A B C$ no ambiente hospitalar. Este número foi evoluindo ao longo do tempo para atingir o ápice em 2003 com 38 publicações. Naquele mesmo ano, Azevedo, Santos e Pamplona (2004), já identificaram queda no uso pragmático da abordagem por parte das maiores empresas brasileiras.

Além dos estudos acima, existem importantes contribuições que não estão registradas na Plataforma Lattes, como: Azevedo, Santos e Pamplona (2004); Falk (2001); Khoury (1999) e Martins (2000), dentre outros.

$A$ evolução da pesquisa sobre o Custeio $A B C$, no ambiente hospitalar, consubstancia-se em um forte sinalizador de aplicabilidade da metodologia para apuração dos custos hospitalares. No entanto, isso só pode ser realmente afirmado se averiguado empiricamente. Diante disso, pretende-se, neste estudo, reaplicar a pesquisa realizada por Khoury (1999) nos hospitais universitários e de ensino brasileiros. O objetivo é verificar o uso do Custeio $A B C$ nos hospitais universitários e de ensino brasileiros e comparar os resultados com as pesquisas realizadas anteriormente.

Tabela 1 Produção Científica - Custeio ABC

\begin{tabular}{l|c|c|c|c|c|c|c|c|c|c|c} 
Categoria & $\mathbf{1 9 9 6}$ & $\mathbf{1 9 9 7}$ & $\mathbf{1 9 9 8}$ & $\mathbf{1 9 9 9}$ & $\mathbf{2 0 0 0}$ & $\mathbf{2 0 0 1}$ & $\mathbf{2 0 0 2}$ & $\mathbf{2 0 0 3}$ & $\mathbf{2 0 0 4}$ & $\mathbf{2 0 0 5}$ & Totais \\
Anais & 0 & 0 & $\mathbf{2}$ & $\mathbf{2}$ & $\mathbf{2}$ & 3 & 10 & 19 & 9 & 9 & 56 \\
\hline Periódicos & 1 & 0 & 0 & 0 & 0 & 2 & 2 & 6 & 4 & 5 & 20 \\
\hline Teses & 0 & 0 & 0 & 0 & 0 & 0 & 0 & 0 & 1 & 1 & 2 \\
\hline Dissertações & 0 & 0 & 0 & 2 & 4 & 1 & 2 & 11 & 3 & 5 & 28 \\
\hline Monografias & 0 & 0 & 0 & 0 & 3 & 0 & 4 & 2 & 6 & 6 & 21 \\
\hline Livros & 0 & 0 & 0 & 0 & 0 & 1 & 0 & 0 & 0 & 0 & 1 \\
\hline Capítulos de Livros & 0 & 0 & 0 & 0 & 0 & 2 & 0 & 0 & 0 & 0 & 2 \\
\hline Totais & 1 & 0 & 2 & 4 & 9 & 9 & 18 & 38 & 23 & 26 & 130
\end{tabular}




\section{METODOLOGIA}

Com base nos objetivos, o trabalho classifica-se como uma pesquisa descritiva, ou seja, aquela que tem como objetivo principal a descrição das características de determinada população ou fenômeno, estabelecendo relações entre as variáveis. Quanto à abordagem do problema, o estudo classifica-se como uma pesquisa quantitativa. Como o próprio nome diz, essas pesquisas se caracterizam pelo emprego de instrumentos estatísticos, tanto na coleta quanto no tratamento dos dados. Quanto aos procedimentos técnicos de coleta e análise de dados, o estudo foi realizado por meio de um levantamento. Segundo Gil (2002, p. 30), as pesquisas, assim realizadas, são marcadas

[...] pela interrogação direta das pessoas cujo comportamento se deseja conhecer. Basicamente, procedese à solicitação de informações a um grupo significativo de pessoas [amostra] acerca do problema estudado para, em seguida, mediante análise quantitativa, obterem-se as conclusões correspondentes aos dados coletados.

As conclusões acerca do grupo estudado são generalizadas para toda a população, mediante uma margem de erro preestabelecida. As pesquisas de levantamento, geral- mente, são mais utilizadas em estudos descritivos, cujos resultados não demandam exigência de se aprofundar no problema de pesquisa.

A população pesquisada é composta pelos hospitais filiados à Associação Brasileira de Hospitais Universitários e de Ensino (ABRAHUE), totalizando 115 instituições. De acordo com a ABRAHUE, apenas 22 hospitais não estavam filiados a ela em Agosto/06, período em que foram aplicados os questionários.

Embora o retorno das respostas seja, aparentemente, significativo, foi feito o teste estatístico das proporções, com a finalidade de certificar a representatividade da amostra. Para a realização do teste foi utilizado o percentual de hospitais de acordo com a esfera administrativa (Federal, Estadual e Particular)'. A hipótese nula considera que a proporção dos hospitais respondentes é igual ao universo pesquisado, enquanto a hipótese alternativa considera que a referida proporção é diferente.

Utilizou-se para o nível de significância estatístico $\alpha=$ 0,05 . Para $K=2$ graus de liberdade, tem-se que $X_{0,05}^{2}=$ 5,99 . Após a realização do teste, obteve-se o qui-quadrado $=4,14$. Como $X^{2}>X_{\alpha}^{2}$ a hipótese nula é aceita, ou seja, a amostra é representativa.

\section{A PESQUISA}

\section{1 Perfil da amostra}

Foram enviados questionários, via e-mail, aos 115 hospitais universitários e de ensino filiados à ABRAHUE, no mês de Agosto de 2006. Para facilitar as respostas, os questionários foram compostos por 20 questões fechadas. Obtiveram-se 34 retornos, o equivalente a $30 \%$ dos questionários enviados. A Tabela $2 \mathbf{O}$ apresenta o nível de resposta dos hospitais por esfera administrativa.

Verifica-se que a freqüência de respostas foi maior entre os hospitais federais, obtendo-se $53 \%$ da amostra total. Os demais segmentos tiveram quantidades de respostas menores. No entanto, houve representatividade significativa em todas as esferas administrativas. De forma geral, a participação de cada categoria no total da amostra, em termos percentuais, segue de perto a sua participação na população pesquisada.

No tocante aos níveis de saúde de atuação das instituições, foi constatado que $26 \%$ delas atuam com procedimentos de nível básico e média complexidade e $74 \%$ realizam, também, procedimentos de alta complexidade. Esses são procedimentos de custos extremamente elevados, como é o caso de diálise, da quimioterapia, da radioterapia e da hemo-

Tabela 2 Esfera administrativa dos Hospitais

\begin{tabular}{l|r|r|c|c}
\multirow{2}{*}{ Esfera Administrativa } & \multicolumn{2}{|c|}{ População } & \multicolumn{2}{c}{ Respostas } \\
\cline { 2 - 5 } & Qtde. & Qtde. \% & Qtde. & Qtde. \% \\
\hline Federal & 46 & $40 \%$ & 18 & $53 \%$ \\
\hline Estadual & 32 & $28 \%$ & 7 & $21 \%$ \\
\hline Municipal & 5 & $4 \%$ & 2 & $6 \%$ \\
\hline Particular & 32 & $28 \%$ & 7 & $21 \%$ \\
\hline Total & $\mathbf{1 1 5}$ & $\mathbf{1 0 0 \%}$ & $\mathbf{3 4}$ & $\mathbf{1 0 0 \%}$
\end{tabular}

Fonte: Elaborada pelos autores

\footnotetext{
1 Nos hospitais municipais obteve-se uma taxa de retorno de $40 \%$, ou seja, dois hospitais. Levando-se em consideração que o cálculo das estatísticas de teste qui-quadrado exige uma freqüência igual ou superior a cinco para serem aproximadas por uma distribuição normal de probabilidade, os referidos hospitais foram excluídos do teste. O que não invalida o teste para os demais.
} 
terapia, dentre outros realizados por poucos hospitais. Essa informação denota o porte dos hospitais pesquisados.

Outra característica relacionada ao tamanho das instituições pesquisadas é o número de leitos existentes. De acordo com a Tabela $3 \mathbf{0}$, não houve grande concentração em uma categoria específica. No entanto, verifica-se que $64 \%$ da amostra possui mais de 200 leitos.

\subsection{Perfil dos respondentes}

Os respondentes da pesquisa ocupavam os seguintes cargos nas instituições: $27 \%$ eram diretores; $38 \%$ gerentes; $6 \%$ contadores e $29 \%$ ocupavam outros cargos. Com relação ao tempo em que os respondentes ocupavam os cargos atuais, verificou-se que: $12 \%$ deles estavam no cargo há menos de um ano; $59 \%$ estavam no cargo atual em períodos compreendidos entre um e cinco anos e $29 \%$ deles estavam no cargo há mais de cinco anos.

No tocante à formação acadêmica, foi constatado que todos são graduados, verificando-se que 65\% têm Especialização ou MBA; $3 \%$ têm Mestrado e 6\% têm Doutorado, conforme demonstra a Tabela $4 \mathbf{0}$.

\subsection{A acurácia do Sistema de Custo adotado pelos Hospitais}

A maioria dos hospitais, $65 \%$, entende que possui sistemas de custos razoavelmente acurados e apenas $9 \%$ afirma possuir sistemas muito acurados, como apresenta a Tabela 50

Na pesquisa realizada por Khoury (1999), os percentuais encontrados foram um pouco diferentes: $54 \%$ das empresas assinalaram a opção "razoavelmente acurados" e $44 \%$ afirmaram que seus sistemas de custos eram muito acurados. Na pesquisada realizada nas maiores empresas catarinenses (BEUREN e ROEDEL, 2002), 47\% das empresas afirmaram possuir sistemas de custos razoavelmente acurados e $42 \%$ sistemas de custos muito acurados.

Os dados acima indicam que os sistemas de custos existentes nos hospitais são menos acurados que os existentes nas maiores empresas brasileiras e catarinenses, respectivamente. Essa informação encontra ressonância na literatura do setor, quando se considera a complexidade do ambiente hospitalar. Segundo Drucker (1975), "poucos processos industriais são tão complicados quanto o hospi-

Tabela 3 Quantidade de leitos dos Hospitais

\begin{tabular}{l|c|c}
\hline Alternativas de respostas & $\mathbf{N}^{\circ}$ Hospitais & $\%$ \\
\hline Até 100 & 3 & $9 \%$ \\
\hline De 101 a 200 & 7 & $21 \%$ \\
\hline De 201 a 300 & 11 & $32 \%$ \\
\hline De 301 a 400 & 2 & $6 \%$ \\
\hline Acima de 400 & 9 & $26 \%$ \\
\hline Não disponível & 2 & $6 \%$ \\
\hline Total & $\mathbf{3 4}$ & $\mathbf{1 0 0 \%}$
\end{tabular}

Fonte: Elaborada pelos autores

Tabela 4 | Formação acadêmica dos respondentes

\begin{tabular}{l|c|c}
\multicolumn{1}{c|}{ Alternativas de respostas } & $\mathbf{N}^{\circ}$ Hospitais & $\mathbf{\%}$ \\
\hline Graduação & 9 & $26 \%$ \\
\hline Especialização (Lato Sensu) & 18 & $53 \%$ \\
\hline MBA (Master Business Adminitration) & 4 & $12 \%$ \\
\hline Mestrado (Stricto Sensu) & 1 & $3 \%$ \\
\hline Doutorado (Stricto Sensu) & 2 & $6 \%$ \\
\hline Total & $\mathbf{3 4}$ & $\mathbf{1 0 0 \%}$
\end{tabular}

Fonte: Elaborada pelos autores

Tabela 5 - Acurácia do Sistema de Custos adotado pelos Hospitais

\begin{tabular}{l|c|c} 
Alternativas de respostas & $\mathbf{N}^{\circ}$ Hospitais & $\%$ \\
\hline Muito inacurado & 2 & $6 \%$ \\
\hline Razoavelmente inacurado & 1 & $3 \%$ \\
\hline Razoavelmente acurado & 22 & $65 \%$ \\
\hline Muito acurado & 3 & $9 \%$ \\
\hline Não disponível & 6 & $18 \%$ \\
\hline Total & $\mathbf{3 4}$ & $\mathbf{1 0 0 \%}$
\end{tabular}

Fonte: Elaborada pelos autores 
tal". Tal complexidade também é destacada por Falk (2001, p. 16). Segundo ele,

O controle de custos [...] tem recebido pouco espaço na literatura e, quando encontrado, está dedicado mais aos custos do ambiente industrial. Todavia, sa-

bendo que o hospital é uma das organizações mais complexas de prestação de serviços, temos que procurar afiliar-nos com aqueles que já passaram pelo caminho da floresta e estão no descampado em que há um pouco mais de luz esclarecedora sobre o assunto. (grifo nosso)

Assim, a acurácia dos sistemas de custos dos hospitais, relativamente menor que das maiores empresas brasileiras, aliada à complexidade das atividades realizadas, apontam para a necessidade de sistemas de custos que atendam melhor à apuração dos custos.

\subsection{Importância do Sistema de Custos para a tomada de decisões}

Entre as respostas obtidas, $44 \%$ entendem que o sistema de custo é muito importante, de alta prioridade; $41 \%$ acham que é importante, mas não de alta prioridade. A Tabela $6 \mathbf{0}$ apresenta os resultados.

Essa pergunta não aparece na pesquisa de Khoury (1999). Mas, nas maiores empresas catarinenses, os percentuais dessa questão também foram diferentes. De acordo com a pesquisa, $75 \%$ das empresas entendem que os sistemas de custos são muito importantes, de alta prioridade para a tomada de decisões e $23 \%$ acreditam que eles são importantes, mas não de alta prioridade (BEUREN e ROEDEL, 2002).

A divergência de opiniões entre as maiores empresas catarinenses e os hospitais sobre a importância dos sistemas de custos para a tomada de decisões pode ter como causa o fato de que a maioria absoluta, $79 \%$, são hospitais públicos (Tabela 2). A ênfase sobre a geração de resultados positivos nas instituições públicas é significativamente menor que em instituições particulares. Apenas 21\% dos hospitais pesquisados são particulares e, dentre eles, existem fundações e entidades filantrópicas, nas quais o lucro também não é a meta principal.

Os resultados das duas questões (3.4) e (3.5) estão relacionados, pois é de esperar que sistemas menos acurados também sejam menos valorizados por parte dos gestores. E o contrário também é verdadeiro; se os sistemas não são considerados importantes, por parte da administração, não receberão a atenção necessária para se tornarem cada vez mais acurados.

\subsection{Importância do Sistema de Custos para controle de gastos}

Essa questão não foi utilizada nas pesquisas realizadas nas maiores empresas brasileiras e nem nas maiores empresas catarinenses. No entanto, acredita-se que seja relevante, pois uma das funções básicas da contabilidade de custos é o controle de gastos.

Os resultados encontrados foram razoavelmente diferentes se comparados aos da questão anterior. De acordo com as respostas na Tabela $7 \boldsymbol{\nabla}, 53 \%$ dos hospitais entendem que, para controle de gastos, os sistemas de custos são muito importantes, de alta prioridade e $32 \%$ mencionaram que os sistemas de custos são importantes, mas não de alta prioridade.

\subsection{Como são controlados os Custos Indiretos do Hospital}

Duas empresas assinalaram duas alternativas para essa questão, tornando a totalização maior que 100\%. De acordo

Tabela 6 Importância do Sistema de Custos para tomada de decisões

\begin{tabular}{l|c|c}
\multicolumn{1}{c|}{ Alternativas de respostas } & $\mathbf{N}^{\circ}$ Hospitais & $\%$ \\
\hline Muito importante, alta prioridade & 15 & $44 \%$ \\
\hline Importante, mas não de alta prioridade & 14 & $41 \%$ \\
\hline Sem importância, raramente usado & 2 & $6 \%$ \\
\hline Sem importância, nunca usado & 0 & $0 \%$ \\
\hline Não disponível & 3 & $9 \%$ \\
\hline Total & $\mathbf{3 4}$ & $\mathbf{1 0 0} \%$
\end{tabular}

Fonte: Elaborada pelos autores

Tabela 7 || Importância do Sistema de Custos para controle de gastos

\begin{tabular}{l|c|c}
\multicolumn{1}{c|}{ Alternativas de respostas } & $\mathbf{N}^{\circ}$ Hospitais & $\mathbf{\%}$ \\
Muito importante, alta prioridade & 18 & $53 \%$ \\
\hline Importante, mas não de alta prioridade & 11 & $32 \%$ \\
\hline Sem importância, raramente usado & 3 & $9 \%$ \\
\hline Sem importância, nunca usado & 0 & $0 \%$ \\
\hline Não disponível & 2 & $6 \%$ \\
\hline Total & $\mathbf{3 4}$ & $\mathbf{1 0 0 \%}$
\end{tabular}

Fonte: Elaborada pelos autores 
com a Tabela $8 \boldsymbol{0}$, apenas $27 \%$ dos hospitais controlam os custos indiretos por meio da comparação com o orçamento. A metade das instituições pesquisadas, $50 \%$, realiza o controle, comparando-o com o último período. E um percentual significativo, $26 \%$, não tem qualquer tipo de controle.

Entre as maiores empresas catarinenses, $54 \%$ fazem o controle dos custos indiretos por meio da comparação com o orçamento, $40 \%$ por meio da comparação com o último período e apenas $2 \%$ não têm qualquer tipo de controle (BEUREN e ROEDEL, 2002).

Para Horngren, Foster e Datar (2000, p.135), controle é "o grau de influência que determinado gerente possui sobre os custos, receitas e outros assuntos em causa". Martins (2003, p. 305) afirma que "controlar significa conhecer a realidade, compará-la com o que deveria ser, tomar conhecimento rápido das divergências e suas origens e tomar atitudes para correção". Portanto, a grande finalidade do controle é possibilitar o conhecimento dos desvios e, conseqüentemente, tomar as decisões necessárias. Para tanto, o planejamento do futuro é imprescindível.

Embora 85\% da amostra considerem o sistema de custos importante para tomada de decisão e para controle de gastos (seções 3.4 e 3.5), fica evidente, nessa questão, a carência dos hospitais por sistemas de planejamento e controle. Percebe-se, assim, que mais de um quarto da amostra (26\%) não possui qualquer tipo de controle sobre os custos indiretos; a metade realiza o controle por meio da comparação com o último período, ou seja, não há planejamento e pouco mais de um quarto dos respondentes (27\%) controlam os custos indiretos comparando com o orçamento. Ainda assim, não se sabe como é elaborado o orçamento, se é uma simples repetição do passado ou se há, efetivamente, o envolvimento de todas as áreas do hospital.

\subsection{Experiência dos Hospitais com relação ao Custeio $A B C$}

A Tabela 90 apresenta a experiência dos hospitais com relação ao Sistema de Custos $A B C$. Em seguida, são analisados os resultados de cada uma das alternativas e comparados com as outras pesquisas realizadas no cenário brasileiro: Khoury,(1999); Beuren e Roedel (2002) e Azevedo, Santos e Pamplona (2004), demonstrados na Tabela 10.

Sobre o conhecimento do Custeio Baseado em Atividades, foi verificado que a abordagem está bastante disseminada no ambiente hospitalar, pois somente $3 \%$ da amostra declararam nunca ter ouvido falar sobre o Custeio $A B C$. Esse percentual foi o menor encontrado entre as pesquisas brasileiras realizadas, conforme a Tabela 100 .

Apenas 15\% das instituições hospitalares pesquisadas afirmaram não possuir interesse em adotar o Sistema de Custos $A B C$. Esse percentual também foi o menor entre as pesquisas realizadas. Por outro lado, a alternativa "Estão avaliando" foi a que teve o maior percentual, $44 \%$ dos respondentes marcaram essa opção. Esse resultado também foi maior que os resultados apurados nas pesquisas precedentes. Isso leva a crer que há uma expectativa por parte dos hospitais de que o Custeio $A B C$ venha a atender as suas necessidades.

O percentual de instituições que decidiram não utilizar o Custeio $A B C$ é o maior entre todas as pesquisas, $24 \%$ dos hospitais assinalaram essa alternativa. Nas pesquisas realizadas nas maiores empresas brasileiras, esse percentual foi de $9 \%, 15 \%$ e $20 \%$, respectivamente.

$O$ percentual de entidades que decidiram utilizar o Custeio $A B C$ e que estão implantando ou utilizando foi de $15 \%$. Somente na pesquisa realizada em por Khoury(1999), houve um percentual superior (22\%), embora

Tabela 8 | Como são controlados os Custos Indiretos dos Hospitais

\begin{tabular}{l|c|c}
\multicolumn{1}{c|}{ Alternativas de respostas } & $\mathbf{N}^{\circ}$ Hospitais & $\%$ \\
Comparação com o orçamento & 4 & $12 \%$ \\
\hline Comparação com o orçamento flexível & 5 & $15 \%$ \\
\hline Comparação com o último período & 17 & $50 \%$ \\
\hline Sem controle & 9 & $26 \%$ \\
\hline Não disponível & 1 & $3 \%$
\end{tabular}

Fonte: Elaborada pelos autores

Tabela 9 || Experiência dos Hospitais com relação ao Custeio ABC

\begin{tabular}{l|c|c}
\multicolumn{1}{c|}{ Alternativas de respostas } & $\mathbf{N}^{\circ}$ Hospitais & $\%$ \\
Nunca ouviram falar & 1 & $3 \%$ \\
\hline Não se interessam & 5 & $15 \%$ \\
\hline Estão avaliando & 15 & $44 \%$ \\
\hline Decidiram não utilizar & 8 & $24 \%$ \\
\hline Decidiram utilizar, estão implantando ou utilizando & 5 & $15 \%$ \\
\hline Decidiram abandonar & 0 & $0 \%$ \\
\hline Total & $\mathbf{3 4}$ & $\mathbf{1 0 0 \%}$
\end{tabular}

Fonte: Elaborada pelos autores 
Tabela 10 || Pesquisas brasileiras sobre a experiência das empresas com Custeio $A B C$

\begin{tabular}{l|r|r|r|r}
\multicolumn{1}{c|}{ Alternativas de respostas } & $\mathbf{1 9 9 9}$ & $\mathbf{2 0 0 2}$ & $\mathbf{2 0 0 4}$ & $\mathbf{2 0 0 6}$ \\
Nunca ouviram falar & $7 \%$ & $7 \%$ & $12 \%$ & $3 \%$ \\
\hline Não se interessam & $34 \%$ & $58 \%$ & $26 \%$ & $15 \%$ \\
\hline Estão avaliando & $28 \%$ & $5 \%$ & $26 \%$ & $44 \%$ \\
\hline Decidiram não utilizar & $9 \%$ & $15 \%$ & $20 \%$ & $24 \%$ \\
\hline Decidiram utilizar, estão implantando ou utilizando & $22 \%$ & $12 \%$ & $14 \%$ & $15 \%$ \\
\hline Decidiram abandonar & $1 \%$ & $2 \%$ & $3 \%$ & $0 \%$ \\
\hline Totais & $\mathbf{1 0 0} \%$ & $\mathbf{1 0 0 \%}$ & $\mathbf{1 0 0 \%}$ & $\mathbf{1 0 0 \%}$
\end{tabular}

Fonte: Elaborada pelos autores

não tenha havido nenhuma empresa do setor de serviços. Nas demais pesquisas, os resultados foram menores, $12 \%$, em 2002 e 14\%, em 2004.

Diferentemente do ocorrido nas outras pesquisas, nenhum hospital que tenha implantado o Custeio $A B C$ decidiu abandoná-lo. Isso é positivo, pois indica boa aceitação dos hospitais, e embora, é importante relembrar, a abordagem seja relativamente nova no ambiente hospitalar.

A partir dos resultados dessa questão, pode-se inferir que os hospitais universitários e de ensino apresentam expectativas otimistas com relação ao Custeio $A B C$. Talvez a complexidade do ambiente hospitalar tenha se convertido em um aspecto positivo, pois o uso tardio do Custeio $A B C$ no ambiente hospitalar pode ter possibilitado que houvesse mais estudos e, conseqüentemente, evoluções na abordagem.

Aos hospitais que marcaram a alternativa "Não se interessam", foram questionadas quais as razões pelas quais a instituição não se interessou em utilizar o Custeio $A B C$. De acordo com a Tabela $11 \boldsymbol{0}$, a complexidade do Sistema de Custos $A B C$ é a principal causa da falta de interesse. As outras causas mencionadas são: a existência de outras prioridades e o sistema atual atende às necessidades do hospital.

Nas pesquisas realizadas por Khoury (1999) e Beuren e Roedel (2002), a principal razão apontada como causa do não interesse das empresas em utilizar o Custeio $A B C$ foi "o sistema utilizado atende às necessidades da empresa". A segunda razão apresentada foi a complexidade do Sistema $A B C$. Portanto, não houve divergência significativa entre as pesquisas.

Também foram questionadas aos hospitais que decidiram não utilizar o Custeio $A B C$, quais seriam as razões para tal decisão. Na Tabela $12 \boldsymbol{\bullet}$, estão apresentados os princi- pais motivos. Muitas instituições assinalaram mais de uma alternativa, portanto, a totalização é maior que $100 \%$.

As principais razões apresentadas foram: a estrutura atual de informações dos hospitais, apontada por $75 \%$ dos hospitais e a complexidade de implantação e operação do Sistema $A B C$, mencionada por $63 \%$ das instituições que decidiram não utilizar o Custeio $A B C$.

\subsection{Características motivadoras da implantação do Sistema ABC}

Aos hospitais que decidiram utilizar o modelo e estavam implantando ou utilizando o sistema de Custeio Baseado em Atividades, foram questionadas quais seriam as razões que os levaram a implantar o referido sistema. Como alguns hospitais marcaram mais de uma opção, a totalização é maior que $100 \%$. As principais razões, apresentadas na Tabela $13 \boldsymbol{\theta}$, foram "discernimento das causas do aumento ou redução de custos" e "custos mais acurados para precificação".

É interessante notar que "análise do lucro mais acurada para tomada de decisões" não foi assinalada por nenhum hospital. Na pesquisa realizada por Beuren e Roedel (2002), foi a alternativa mais escolhida pelos respondentes: $57 \%$ da amostra. E na pesquisa realizada por Khoury (1999), essa foi a segunda alternativa mais escolhida, apontada por $56 \%$ das empresas. A pouca importância atribuída à acurácia da informação "lucro" por parte dos hospitais torna-se compreensível quando se verifica que $79 \%$ da amostra é constituída por hospitais públicos e apenas $21 \%$ particulares, sendo que dentre esses existem fundações e entidades filantrópicas. Como já foi mencionado, o lucro com os procedimentos médicos pode não ser a meta principal de tais empreendimentos.

Tabela 11 Razões pelas quais as Instituições não se interessam em utilizar o Custeio ABC

\begin{tabular}{l|c|c}
\multicolumn{1}{c|}{ Alternativas de respostas } & $\mathbf{N}^{\circ}$ Hospitais & $\%$ \\
Os istema utilizado atende às necessidades do hospital & 1 & $20 \%$ \\
\hline A utilização do Sistema ABC não é prioritária & 1 & $20 \%$ \\
\hline Complexidade de implantação e operação do Sistema ABC & 2 & $40 \%$ \\
\hline Outra & 1 & $20 \%$ \\
\hline Total & $\mathbf{5}$ & $\mathbf{1 0 0 \%}$
\end{tabular}

Fonte: Elaborada pelos autores 
Tabela 12 Razões pelas quais os Hospitais decidiram não utilizar o Custeio ABC

\begin{tabular}{l|c|c}
\multicolumn{1}{c|}{ Alternativas de respostas } & No Motivos & $\%$ \\
A estrutura das informações não está de acordo com o ABC & 6 & $75 \%$ \\
\hline Complexidade de implantação e operação do Sistema ABC & 5 & $63 \%$ \\
\hline A utilização do Sistema ABC não é prioritária & 2 & $25 \%$ \\
\hline Relação custo versus benefício & 2 & $25 \%$ \\
\hline Devido à estrutura de custos do hospital & 2 & $25 \%$ \\
\hline Alto custo e benefícios duvidosos & 1 & $13 \%$ \\
\hline Orientação da direção do hospital & 1 & $13 \%$ \\
\hline O Sistema ABC não é adequado para este negócio & 1 & $13 \%$ \\
\hline Falta de profundo conhecimento do Sistema ABC & 1 & $13 \%$ \\
\hline Possibilidade de vir a ser implantado no futuro & 1 & $13 \%$ \\
\hline Necessidade de um sistema paralelo & 1 & $13 \%$ \\
\hline Outras & 2 & $25 \%$
\end{tabular}

Fonte: Elaborada pelos autores

Tabela 13 | Características motivadoras da implantação do Sistema ABC

\begin{tabular}{l|c|c}
\multicolumn{1}{c|}{ Alternativas de respostas } & $\mathbf{N}^{\circ}$ Hospitais & $\%$ \\
Dicernimento das causas do aumento ou redução de custos & 3 & $60 \%$ \\
\hline Custos mais acurados para precificação & 3 & $60 \%$ \\
\hline Análise dos custos dos estoques & 2 & $40 \%$ \\
\hline Melhoria nos processos e práticas de produção e marketing & 1 & $20 \%$ \\
\hline Análise do lucro mais acurada para tomada de decisão & 0 & $0 \%$
\end{tabular}

Fonte: Elaborada pelos autores

\subsection{Tempo de implantação do Sistema ABC}

Seguindo a tendência apurada nas outras pesquisas, a implantação do Sistema $A B C$ nos hospitais também é recente. Como apresentado na Tabela $14 \boldsymbol{O}$, apenas um hospital possui o sistema implantado num período superior a dois anos. Há um hospital, não mencionado na Tabela 14 , no qual o sistema está em fase de implantação.

\subsection{O Sistema ABC e os Sistemas existentes no Hospital}

Em nenhum dos hospitais que adotaram o Sistema $A B C$, os sistemas tradicionais foram totalmente substituídos. Em dois deles, em que o Sistema $A B C$ foi recentemente implantado, ainda não foi decidido sobre a possível substituição; a decisão ocorrerá no futuro. Nos demais, o
Sistema ABC suplementou, de alguma forma, os sistemas já existentes. A Tabela $15 \bullet$ apresenta os percentuais.

A Tabela $16 \bullet$ apresenta a comparação entre os hospitais e as grandes empresas (pesquisas disponíveis) no que se refere à suplementação ou substituição de outros sistemas por parte do Sistema $A B C$.

Na pesquisa realizada por Khoury (1999), foi verificado que, em $64 \%$ dos casos, o Sistema $A B C$ suplementou os sistemas existentes, um percentual superior ao apurado nessa pesquisa, que foi de $40 \%$. Nas maiores empresas catarinenses, houve bastantes substituições: em $42 \%$ das empresas que adotaram o Custeio Baseado em Atividades, os sistemas anteriores deixaram de existir. Nesta pesquisa, a alternativa ficou zerada, isso ocorreu porque o Sistema $A B C$ é relativamente novo no ambiente hospitalar e os gestores ainda não estão

Tabela 14 Tempo de implantação do Sistema ABC

\begin{tabular}{l|c|c} 
Alternativas de respostas & $\mathbf{N}^{\circ}$ Hospitais & $\mathbf{\%}$ \\
Menos de seis meses & 1 & $25 \%$ \\
\hline Entre seis meses e um ano & 1 & $25 \%$ \\
\hline Entre um e dois anos & 1 & $25 \%$ \\
\hline Entre dois e três anos & 0 & $0 \%$ \\
\hline Mais de três anos & 1 & $25 \%$ \\
\hline Total & $\mathbf{4}$ & $\mathbf{1 0 0} \%$
\end{tabular}

Fonte: Elaborada pelos autores 
Tabela 15 | O Sistema ABC suplementou ou substituiu o Sistema de Custos Tradicional (Hospitais)

\begin{tabular}{l|c|c}
\multicolumn{1}{c|}{ Alternativas de respostas } & $\mathbf{N}^{\circ}$ Hospitais & $\%$ \\
Suplementou & 2 & $40 \%$ \\
\hline Substituiu & 0 & $0 \%$ \\
\hline Suplementou ou substituiu em algumas partes & 1 & $20 \%$ \\
\hline Será decidido futuramente & 2 & $40 \%$ \\
\hline Total & $\mathbf{5}$ & $\mathbf{1 0 0 \%}$
\end{tabular}

Fonte: Elaborada pelos autores

Tabela 16 O Sistema ABC suplementou ou substituiu o Sistema de Custos Tradicional (pesquisas brasileiras)

\begin{tabular}{l|r|r|r|}
\multicolumn{1}{c|}{ Alternativas de respostas } & $\mathbf{1 9 9 9}$ & $\mathbf{2 0 0 2}$ & $\mathbf{2 0 0 6}$ \\
Suplementou & $64 \%$ & $\mathbf{8} \%$ & $40 \%$ \\
\hline Substituiu & $14 \%$ & $42 \%$ & $0 \%$ \\
\hline Suplementou ou substituiu em algumas & $20 \%$ & $33 \%$ & $20 \%$ \\
\hline Será decidido futuramente & $2 \%$ & $17 \%$ & $40 \%$ \\
\hline Totais & $\mathbf{1 0 0 \%}$ & $\mathbf{1 0 0} \%$ & $\mathbf{1 0 0} \%$ \\
\hline
\end{tabular}

Fonte: Elaborada pelos autores

suficientemente seguros para abandonar totalmente os sistemas tradicionais.

A integração do Sistema $A B C$ com outros sistemas foi verificada apenas no controle de estoques. Três dos hospitais que adotaram o Custeio ABC têm o sistema interligado ao Just-in-time, como apresenta a Tabela $17 \boldsymbol{0}$.

Os percentuais apurados nas outras pesquisas, observando-se a ligação do Sistema $A B C$ com outros sistemas, apresentados na Tabela $18 \boldsymbol{0}$, foram muito diferentes daqueles apurados nos hospitais universitários e de ensino. Na pesquisa realizada por Khoury (1999), os respondentes apontaram mais de uma alternativa, por isso, a totalização é maior que $100 \%$.

A ligação com o Just-in-time, apontada por $60 \%$ dos hospitais, foi assinalada por apenas $2 \%$ na pesquisa de 1996, não tendo sido marcada por nenhuma empresa catarinense. A ligação com o sistema de Administração da Qualidade Total (TQM) não foi mencionada por nenhum hospital; nas outras pesquisas, houve percentuais de $24 \%$ e $25 \%$, respectivamente. A Reengenharia, muito pouco falada nos dias de hoje, aparece apenas em 1996, com 32\% das empresas.

Tabela 17 || O Sistema de Custos $A B C$ está ligado a quais sistemas (Hospitais)

\begin{tabular}{l|c|c}
\multicolumn{1}{c|}{ Alternativas de respostas } & $\mathbf{N}^{\circ}$ Hospitais & $\%$ \\
Just-in-time & 3 & $60 \%$ \\
\hline Administração da Qualidade Total (TQM) & 0 & $0 \%$ \\
\hline Teoria das Restrições & 0 & $0 \%$ \\
\hline Reengenharia & 0 & $0 \%$ \\
\hline Outro. Especifique: & 1 & $20 \%$ \\
\hline Não é ligado a nenhum sistema & 1 & $20 \%$ \\
\hline Total & $\mathbf{5}$ & $\mathbf{1 0 0 \%}$
\end{tabular}

Fonte: Elaborada pelos autores

Tabela 18 O Sistema de Custos $A B C$ está ligado a quais Sistemas (pesquisas brasileiras)

\begin{tabular}{l|r|r|c|}
\multicolumn{1}{c|}{ Alternativas de respostas } & $\mathbf{1 9 9 9}$ & $\mathbf{2 0 0 2}$ & $\mathbf{2 0 0 6}$ \\
Just-in-time & $\mathbf{2 \%}$ & $0 \%$ & $60 \%$ \\
\hline Administração da Qualidade Total (TQM) & $24 \%$ & $25 \%$ & $0 \%$ \\
\hline Teoria das Restrições & $2 \%$ & $0 \%$ & $0 \%$ \\
\hline Reengenharia & $32 \%$ & $0 \%$ & $0 \%$ \\
\hline Outro. Especifique: & $14 \%$ & $25 \%$ & $20 \%$ \\
\hline Não é ligado a nenhum sistema & $42 \%$ & $50 \%$ & $20 \%$ \\
\hline
\end{tabular}

Fonte: Elaborada pelos autores 
Os percentuais de instituições em que o Sistema $A B C$ não estava ligado a nenhum outro sistema também foram significativos: 42\%, em 1999; 50\%, em 2002 e 20\%, em 2006. É de se esperar que, com os avanços do Custeio Baseado em Atividades e dos sistemas de informática aplicados à contabilidade, a integração vá aumentando gradualmente.

\subsection{Posição das pessoas envolvidas no desenvolvimento do Sistema de Custos ABC}

Em $60 \%$ dos hospitais que implantaram o Sistema de Custos $A B C$, havia o envolvimento de pessoas da contro- ladoria e também de técnicos. Conforme apresentado na Tabela 19 D, a alta administração e o pessoal de sistemas estavam envolvidos no desenvolvimento do Sistema $A B C$ em $40 \%$ dos casos de implantação.

Essa questão aparece somente na pesquisa realizada por Khoury (1999), em que o percentual de pessoal da controladoria envolvido no desenvolvimento do Sistema de Custos $A B C$ era muito superior; em $96 \%$ das empresas que adotaram o sistema havia a participação da Controladoria, evidenciando-se menor o envolvimento das demais áreas, como demonstra a Tabela $20 \mathbf{0}$.

Tabela 19 | Posição das pessoas envolvidas no desenvolvimento do Sistema de Custos ABC

\begin{tabular}{l|c|c} 
Alternativas de respostas & $\mathbf{N}^{\circ}$ Hospitais & $\%$ \\
Alta administração & 2 & $40 \%$ \\
\hline Controladoria & 3 & $60 \%$ \\
\hline Técnicos & 3 & $60 \%$ \\
\hline Sistemas & 2 & $40 \%$ \\
\hline Consultoria externa & 1 & $20 \%$ \\
\hline Outro & 1 & $20 \%$
\end{tabular}

Fonte: Elaborada pelos autores

Tabela 20 | Posição das pessoas envolvidas no desenvolvimento do Sistema de Custos ABC (pesquisas brasileiras)

\begin{tabular}{l|r|l|}
\multicolumn{1}{c|}{ Alternativas de respostas } & $\mathbf{1 9 9 9}$ & $\mathbf{2 0 0 6}$ \\
\hline Alta administração & $\mathbf{2 4 \%}$ & $40 \%$ \\
\hline Controladoria & $96 \%$ & $60 \%$ \\
\hline Técnicos & $40 \%$ & $60 \%$ \\
\hline Sistemas & $30 \%$ & $40 \%$ \\
\hline Consultoria externa & $8 \%$ & $20 \%$ \\
\hline Outro & $42 \%$ & $20 \%$ \\
\hline
\end{tabular}

Fonte: Elaborada pelos autores

\section{CONSIDERAÇÕES FINAIS}

O ambiente hospitalar apresenta-se como um local extremamente complexo para a adequada apuração de custos dos procedimentos ali realizados. Isso ocorre porque são vários os tipos de serviços prestados: desde o nascimento do indivíduo, passando por prevenção contra as doenças, até a busca pela cura dos males que afetam, das mais variadas formas, a saúde humana. Além disso, cada organismo reage de uma determinada forma quando submetido a um procedimento médico, requerendo atenção e medicamentos diversos, mesmo em se tratando de males semelhantes. Assim, a padronização dos serviços, extremamente importante na definição de um sistema de custos, é uma tarefa bastante complexa.

O intrincado ambiente hospitalar, em face dos problemas mencionados, não tem recebido a atenção desejável dos estudiosos de custos. É pequena a literatura que trata do assunto. No entanto, nos últimos dez anos, vem crescendo o número de estudos que versam sobre a aplicação do
Custeio Baseado em Atividades no ambiente hospitalar. Porém, a grande maioria desses estudos é de natureza teórica, o que torna a necessidade de conhecer o ambiente pragmático premente. Estudos empíricos sobre o Custeio Baseado em Atividades, em outros segmentos no Brasil, também são poucos. Entre eles, destacam-se: Khoury (1999), Beuren e Roedel (2002) e Azevedo, Santos e Pamplona (2004).

Neste trabalho, a amostra foi composta por 34 hospitais filiados à ABRAHUE, o equivalente a 30\% dos 115 filiados. São hospitais de grande porte, $74 \%$ deles realizam procedimentos básicos, de média e alta complexidade e $64 \%$ possuem mais de 200 leitos.

A pesquisa revelou que os gestores dos hospitais consideram seus sistemas de custos atuais com um grau de acurácia menor que o encontrado nas pesquisas realizadas nas maiores empresas brasileiras. A importância atribuída ao sistema de custos para tomada de decisões também foi menor que nas outras pesquisas. Além disso, somente 27\% 
dos respondentes mencionaram controlar os custos indiretos por meio do orçamento, o restante compara com custos passados ou não controla. Essas informações demonstram quão longe estão os sistemas de custos hospitalares atuais de serem ferramentas úteis para a tomada de decisões.

O Custeio $A B C$ mostrou-se bastante conhecido no ambiente hospitalar: $97 \%$ da amostra já ouviram falar sobre a abordagem. Ainda nessa perspectiva, 44\% dos pesquisados mencionaram estarem avaliando o Custeio Baseado em Atividades, representando potenciais usuários da abordagem. O percentual de empresas que decidiram utilizar o sistema não é muito diferente daqueles encontrados nas demais pesquisas brasileiras: 15\% da amostra estão implantando ou utilizando o ABC. Além disso, nenhum hospital que o tenha implantado, decidiu abandoná-lo.

As principais razões que motivaram os hospitais a implantarem o Custeio $A B C$ foram: o discernimento das causas do aumento ou redução de custos e custos mais acurados para precificação. Contrariando o resultado das demais pesquisas, no ambiente hospitalar não há interesse em utilizar o Sistema de Custos $A B C$ para análise do lucro mais acurada para tomada de decisões, ou seja, o resultado positivo não aparece como uma das principais metas dos hospitais universitários e de ensino.

Embora os estudos sobre o uso do Custeio $A B C$ no ambiente hospitalar tenha se iniciado, no Brasil, há cerca de dez anos, a implantação do referido sistema nos hospitais é relativamente nova, apenas um hospital dentre os pesquisados possui o Custeio $A B C$ implantado há mais de dois anos. $O$
Sistema $A B C$ suplementou os sistemas existentes na maior parte das empresas pesquisadas (64\%) e estava interligado ao Just-in-time em $60 \%$ dos casos. Embora o pessoal da Controladoria dos hospitais estivesse envolvido na implantação do Sistema $A B C$ em $60 \%$ dos hospitais que adotaram a abordagem, esse número é pequeno, se comparado àquele apurado por Khoury (1999), que foi de $96 \%$.

A complexidade, presente tanto no Custeio Baseado em Atividades quanto nas atividades desenvolvidas no ambiente hospitalar, parece, a princípio, uma barreira que se opõe ao uso do Sistema de Custeio $A B C$ nos hospitais. No entanto, como foi verificado empiricamente, isso não ocorre. Talvez, o Custeio ABC, justamente por ser mais complexo, tenha capacidade de interpretar, com maior acurácia, os procedimentos hospitalares. O Custeio $A B C$ consegue analisar detalhes que as abordagens tradicionais ignoram, proporcionando informações economicamente mais acuradas para decisões.

Constitui-se como limitação do presente estudo, o fato de que as comparações foram feitas com pesquisas realizadas em grandes empresas de um modo geral, enquanto este trabalho se restringiu aos hospitais universitários, embora também sejam hospitais de grande porte.

Sugere-se, para trabalhos futuros, um estudo comparativo entre os hospitais que utilizam o Custeio $A B C$, a fim de identificar as semelhanças e diferenças entre os usos da abordagem. Um estudo desta natureza poderia contribuir significativamente na divulgação de experiências entre os hospitais que utilizam e aqueles que desejam utilizar o Custeio $A B C$.

\section{Referências Bibliográficas}

ABRAHUE - Associação Brasileira de Hospitais Universitários e de Ensino. Disponível em: <http://www.abrahue.org.br/>. Acesso em $1^{\circ}$ Ago.2006.

AZEVEDO, Henrique Siqueira dę; SANTOS, Mayara Cristina Seixas; PAMPLONA, Edson de O. Utilização do Sistema ABC no Brasil: Uma Survey entre as 500 Maiores Empresas. Anais do XI Congresso Brasileiro de Custos. Porto Seguro, Bahia, julho de 2004.

BEUREN, Ilse Maria; ROEDEL, Ari. O uso do Custeio Baseado em Atividades - ABC (Activity Based Costing) nas maiores empresas de Santa Catarina. Revista Contabilidade \& Finanças - USP, São Paulo, n. 30, p. 7-18, set./dez. 2002.

DRUंCKER, Peter. Administração: responsabilidades, tarefas e práticas. São Paulo: Pioneira, 1975.

FALK, James Anthony. Gestão de custos para hospitais: conceitos, metodologias e aplicações. São Paulo: Atlas, 2001.

GIL, Antônio Carlos. Como elaborar projeto de pesquisa. 4. ed. São Paulo: Atlas, 2002. 159p.

HORNGREN, Charles T. Contabilidade de Custos. 11ª. ed. São Paulo: Atlas, 2000.

KHOURY, Carlos Yorghi. ABC - sistema de custos baseado em atividades: uma pesquisa de sua utilização no Brasil. RAE - Revista de Administração de Empresas. São Paulo, FGV. v. 39, n. 4, p. 55-63, out./dez. 1999.

MARTINS, Domingos. Custos e Orçamentos Hospitalares. São Paulo: Atlas, 2000.

MARTINS, Eliseu. Contabilidade de Custos. 9. ed. São Paulo: Atlas, 2003.

PLATAFORMA LATTES. Disponível em: <http://lattes.cnpq.br/index.htm>.Acesso em 14.ago.2006.

\section{NOTA - Endereço dos autores}

Universidade Federal de Uberlândia

Faculdade de Ciências Integradas do Pontal

Av. José João Dib, 2545 - Progresso

Ituiutaba - MG

38302-000
Universidade Federal de Uberlândia

Faculdade de Ciências Contábeis

Av. João Naves de Ávila, 2121 - Santa Mônica

Uberlândia-MG

38400-902 